\title{
Exchange Coupling in Heterodinuclear Complexes based on Differential Local Spin Rotations
}

\author{
Rajendra P. Joshi, Jordan J. Phillips, and Juan E. Peralta* \\ Department of Physics and Science of Advanced Materials, Central Michigan University, \\ Mount Pleasant, MI, 48859, USA \\ E-mail: peral1j@cmich.edu
}

*To whom correspondence should be addressed 


\section{Derivation}

Here we derive Eq. 3 from the main text. To this end, we consider two spin centers with maximum possible spin $S_{1}$ and $S_{2}$ which we represent by the ket

$$
|\alpha\rangle=\left|S_{1}, S_{2}\right\rangle=\left|S_{1}\right\rangle\left|S_{2}\right\rangle
$$

If we rotate $S_{2}$ by an angle $\theta$ about y-axis, then $S_{2}$ can be written as

$$
\left|S_{2}\right\rangle=e^{-i \theta S_{y}}\left|S_{2}\right\rangle
$$

The two-spin state $|\alpha\rangle$ can then be expressed as

$$
|\alpha\rangle=\left|S_{1}\right\rangle e^{-i \theta S_{y}}\left|S_{2}\right\rangle
$$

Using this state, we calculate

$$
\frac{\mathrm{d}^{2}\langle\hat{H}\rangle}{\mathrm{d} \theta^{2}}=2\left\langle\alpha^{\prime \prime}|\hat{H}| \alpha\right\rangle+2\left\langle\alpha^{\prime}|\hat{H}| \alpha^{\prime}\right\rangle
$$

where $\left|\alpha^{\prime}\right\rangle=\frac{\mathrm{d}|\alpha\rangle}{\mathrm{d} \theta}$ and $\left|\alpha^{\prime \prime}\right\rangle=\frac{\mathrm{d}^{2}|\alpha\rangle}{\mathrm{d} \theta^{2}}$ are calculated from Eq. 3 which on substituting in Eq. 4 and using operation of raising and lowering operators we derive,

$$
\frac{\mathrm{d}^{2}\langle\hat{H}\rangle}{\mathrm{d} \theta^{2}}=S_{2}\left\langle S_{1} S_{2}-1|\hat{H}| S_{1} S_{2}-1\right\rangle-S_{2}\left\langle S_{1} S_{2}|\hat{H}| S_{1} S_{2}\right\rangle
$$

Using the HDVV Hamiltoninan $\hat{H}=-J \hat{S}_{1} \cdot \hat{S}_{2}$, Eq. 5 at $\theta=0$ can be written as

$$
\left.\frac{\mathrm{d}^{2}\langle\hat{H}\rangle}{\mathrm{d} \theta^{2}}\right|_{\theta=0}=S_{2}\left[-J S_{1}\left(S_{2}-1\right)\right]-S_{2}\left(-J S_{1} S_{2}\right)
$$

which gives 


$$
J=\left.\frac{1}{S_{1} S_{2}} \frac{d^{2}}{d \theta^{2}}\left\langle\hat{H}_{H D V V}\right\rangle\right|_{\theta=0}
$$

\section{Nominal local spin values}

Table 1: Local spin $\mathrm{S}_{1}, \mathrm{~S}_{2}$ on spin centers $\mathrm{M}_{1}, \mathrm{M}_{2}$

\begin{tabular}{lrrrr}
\hline \hline Systems & $\mathrm{M}_{1}$ & $\mathrm{M}_{2}$ & $\mathrm{~S}_{1}$ & $\mathrm{~S}_{2}$ \\
\hline WIXFOZ & $\mathrm{Ni}(\mathrm{II})$ & $\mathrm{VO}(\mathrm{IV})$ & 1 & $1 / 2$ \\
WIXFUF & $\mathrm{Ni}(\mathrm{II})$ & $\mathrm{VO}(\mathrm{IV})$ & 1 & $1 / 2$ \\
PAJZEF & $\mathrm{Cu}(\mathrm{II})$ & $\mathrm{Ni}(\mathrm{II})$ & $1 / 2$ & 1 \\
PAJZAB & $\mathrm{Cu}(\mathrm{II})$ & $\mathrm{Ni}(\mathrm{II})$ & $1 / 2$ & 1 \\
BIGFAY & $\mathrm{Cu}(\mathrm{II})$ & $\mathrm{VO}(\mathrm{IV})$ & $1 / 2$ & $1 / 2$ \\
PUSJOC & $\mathrm{Cu}(\mathrm{II})$ & $\mathrm{VO}(\mathrm{IV})$ & $1 / 2$ & $1 / 2$ \\
\hline \hline
\end{tabular}

\section{Spin density plots}
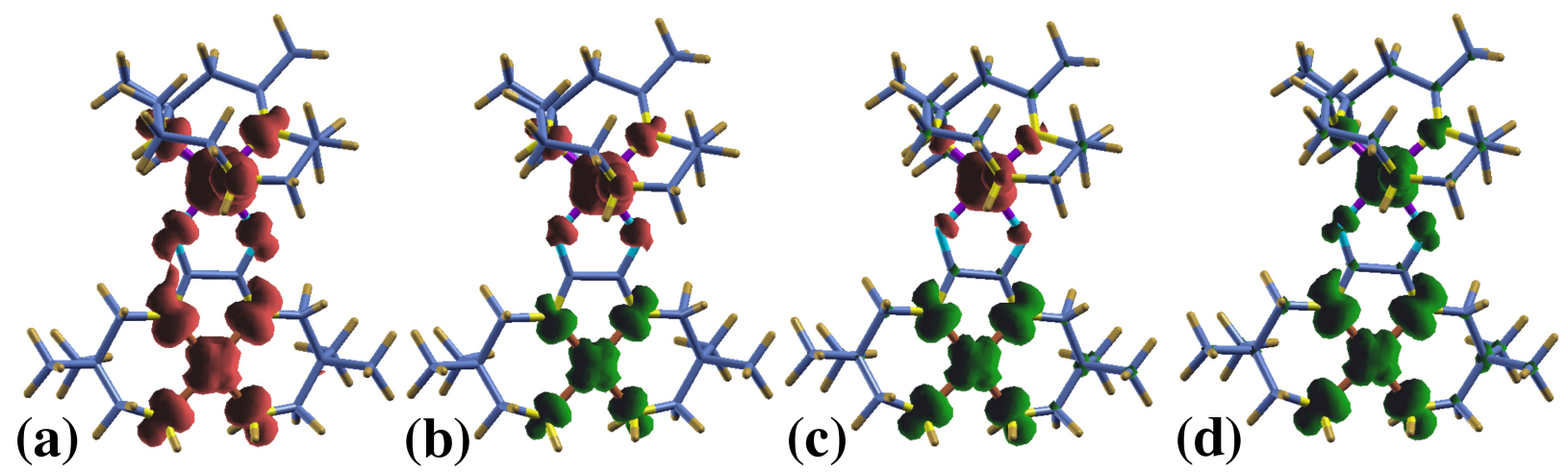

Figure 1: Spin density isosurfaces for the complex PAJZAB. (a) and (b) represent the zero-order spin density (in $\hat{z}$ ) for the HS and BS solutions (isovalue 0.002 a.u.) and (c) and $(\mathrm{d})$ represent the first-order density (in $\hat{x}$ ) (isovalue 1.800 a.u.). Green and red are used for positive and negative isosurfaces, respectively. 

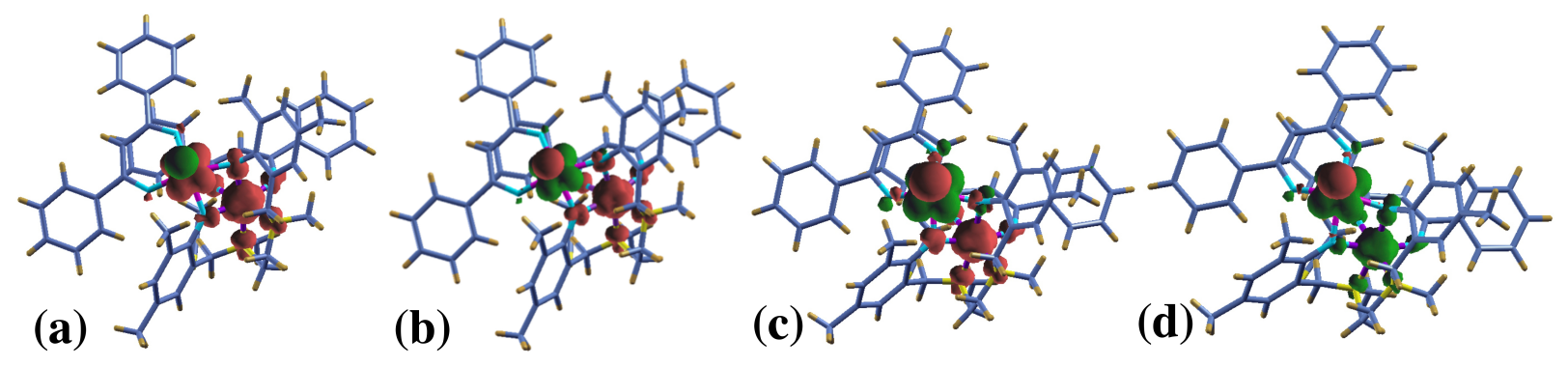

Figure 2: Spin density isosurfaces for the complex WIXFUF. (a) and (b) represent the zero-order spin density (in $\hat{z}$ ) for the HS and BS solutions (isovalue 0.004 a.u.) and (c) and (d) represent the first-order density (in $\hat{x}$ ) (isovalue 18.000 a.u.). Green and red are used for positive and negative isosurfaces, respectively.
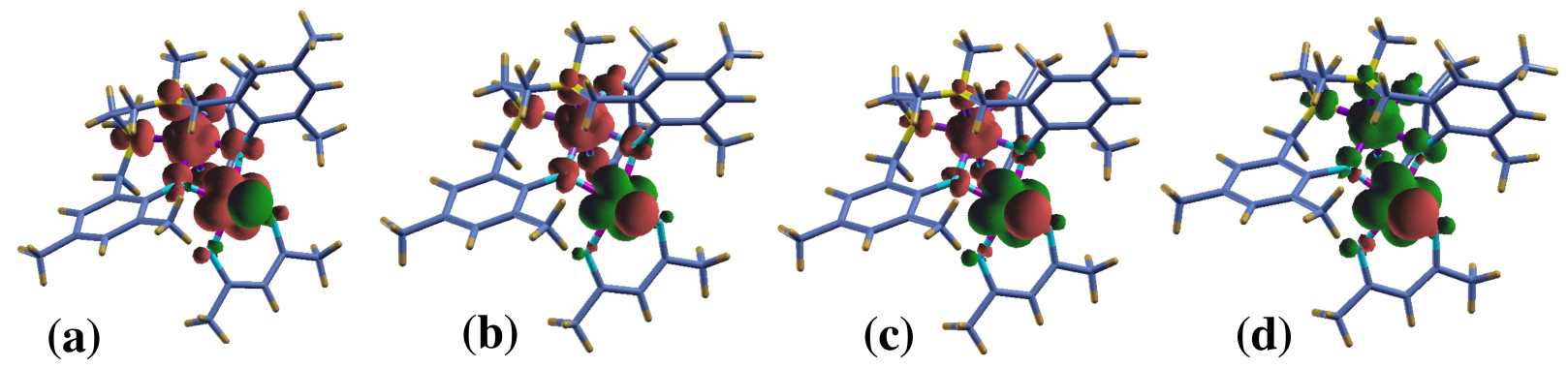

Figure 3: Spin density isosurfaces for the complex WIXFUZ. (a) and (b) represent the zero-order spin density (in $\hat{z}$ ) for the HS and BS solutions (isovalue 0.003 a.u.) and (c) and (d) represent the first-order density (in $\hat{x}$ ) (isovalue 20.000 a.u.). Green and red are used for positive and negative isosurfaces, respectively.

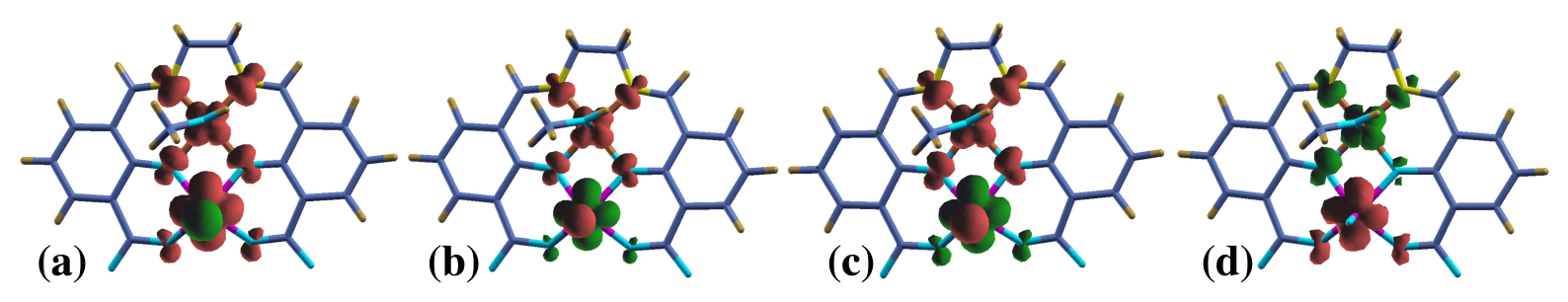

Figure 4: Spin density isosurfaces for the complex BIGFAY. (a) and (b) represent the zero-order spin density (in $\hat{z}$ ) for the HS and BS solutions (isovalue 0.006 a.u.) and (c) and $(\mathrm{d})$ represent the first-order density (in $\hat{x}$ ) (isovalue 14.000 a.u.). Green and red are used for positive and negative isosurfaces, respectively. 

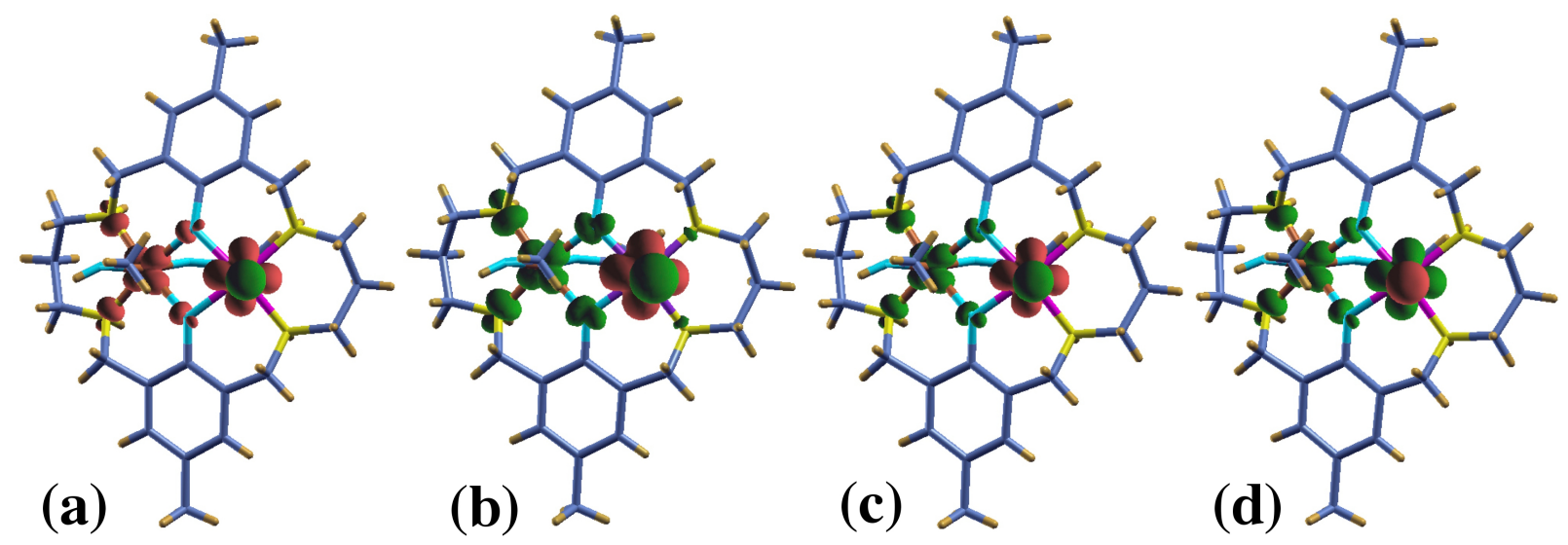

Figure 5: Spin density isosurfaces for the complex PUSJOC. (a) and (b) represent the zero-order spin density (in $\hat{z}$ ) for the HS and BS solutions (isovalue 0.008 a.u.) and (c) and (d) represent the first-order density (in $\hat{x}$ ) (isovalue 21.000 a.u.). Green and red are used for positive and negative isosurfaces, respectively. 